\section{Considerações metodológicas à luz da Pesquisa Mundial de Saúde no Brasil, 2003}

\author{
Methodological notes in light of the \\ World Health Survey in Brazil, 2003
}

\section{Guilherme Loureiro Werneck}

Departamento de Epidemiologia do Instituto de Medicina Social da Universidade do Estado do Rio de Janeiro

Endereço para correspondência: Guilherme Loureiro Werneck. Instituto de Medicina Social/IMS, Departamento de Epidemiologia, Universidade do Estado do Rio de Janeiro (UERJ). Rua São Francisco Xavier, 524 - $7{ }^{\circ}$ andar - Bloco D - Maracanã. 20559-900 Rio de Janeiro/RJ, Brazil. E-mail: gwerneck@ensp.fiocruz.br
O Seminário "Inquéritos Populacionais: Aspectos Metodológicos, Operacionais e Éticos" deixará marcas na trajetória de desenvolvimento da pesquisa epidemiológica brasileira. A escolha de tema tão central para a própria construção do objeto da epidemiologia contribuiu para resgatar a importância deste desenho de estudo para o diagnóstico, monitoramento e avaliação de saúde, e fez justiça ao acúmulo de experiências de pesquisas brasileiras nesta área. Além disso, recolocou no centro do debate metodológico um tipo de estudo que em certas ocasiões chegou a ser relegado a um segundo plano dentro do arsenal de delineamentos epidemiológicos, na medida em que a lógica de uma certa epidemiologia moderna, dos fatores de risco, ocupou os espaços acadêmicos não só no mundo, mas também em muitos dos principais centros de formação epidemiológica no Brasil.

Dentre tantas importantes experiências relatadas neste Seminário, destaca-se a realização, em 2003, da Pesquisa Mundial de Saúde no Brasil (PMS), que pode ser considerada um marco no campo da saúde coletiva brasileira por pelo menos quatro motivos. Primeiro, porque sua concepção se deveu, pelo menos em parte, a uma atuação decisiva de pesquisadores brasileiros da área de saúde coletiva, que se manifestaram criticamente em relação ao modelo original da pesquisa e contribuíram diretamente para definir uma nova metodologia que se deseja com alcance transcultural, mais apropriada para avaliar o desempenho dos sistemas de saúde e prover informações sobre o estado de saúde das populações. Segundo, porque neste processo, e a partir do próprio compromisso com a realização da Pesquisa no Brasil, a comunidade científica brasileira da área de saúde coletiva se colocou no centro do debate internacional sobre estes temas. Terceiro, porque, do ponto de vista teórico-conceitual, a metodologia empregada supera uma visão simplista do problema, incorporando dimensões variadas - desde as relativas à situação de saúde e distribuição dos seus determinantes, até 
aquelas relacionadas ao acesso e cobertura de serviços de saúde, satisfação dos usuários e gastos com saúde - de forma a permitir uma aproximação mais realística, e útil, em consonância com a complexidade desse objeto. Por fim, porque representou, também, um grande esforço para garantir que sua realização fosse pautada em padrões científicos de alto nível, de forma a assegurar a validade de seus resultados e o aval da comunidade científica.

A riqueza desta Pesquisa, traduzida na publicação de uma série de artigos em um suplemento dos Cadernos de Saúde Pública em 2005, desperta também um conjunto de reflexões de cunho metodológico e operacional. Algumas destas inquietações formam o corpo deste texto.

A preocupação com a qualidade do processo de aferição na pesquisa epidemiológica tem sido crescente, com reflexos apreciáveis na produção nacional, como se pode apreender pela cada vez mais freqüente publicação de artigos enfocando desde aspectos relativos à avaliação da confiabilidade e validade de instrumentos em situações específicas, até estudos preocupados com o desenvolvimento e adaptação de questionários. Infelizmente, ao que parece, procedimentos formais de adaptação transcultural dos instrumentos utilizados na PMS não puderam ser realizados. É fato que boa parte das perguntas da PMS já foram informalmente "adaptadas" e amplamente utilizadas em pesquisas nacionais, o que fornece alguma garantia sobre sua aceitabilidade, confiabilidade e validade. Entretanto, é também plausível que, para determinados construtos, como "estado de ânimo" ${ }^{1}$, ou certas abordagens, como no caso das vinhetas ${ }^{2}$, possa ter ocorrido um certo grau de distorção nos resultados. Particularmente em relação ao uso das vinhetas, a falta deste processo de adaptação transcultural pode ter sido um dos motivos para que esta abordagem não tenha sido considerada bem sucedida e útil para a calibração intercultural das respostas sobre auto-avaliação da saúde no âmbito da pes- quisa brasileira ${ }^{2}$. Informação sobre confiabilidade inter-observador e/ou testereteste para algumas questões da PMS, como aquelas referentes à auto-avaliação da saúde ${ }^{3,4}$, incapacidade e limitação funcional $^{5}$ e estado de ânimo ${ }^{1}$, seria também de grande interesse para pesquisadores que eventualmente queiram utilizar alguns dos itens deste questionário.

Merece comentário, também, algumas decisões concernentes às variáveis utilizadas na PMS. Em relação às variáveis empregadas para avaliar desigualdade socioeconômica, os estudos até então divulgados utilizaram fundamentalmente três indicadores - escolaridade, número de bens, gasto domiciliar mensal - que refletem mais "acesso a recursos" do que prestígio social. Afora a escolaridade, que parece ser um indicador relativamente robusto e estável para avaliar a posição socioeconômica ao longo da vida adulta, além de transcender à mera avaliação de poder de compra associando-se com determinados estilos de vida relacionados à saúde, e também refletindo circunstâncias socioeconômicas familiares no início da vida ${ }^{6}$, teria sido oportuna a compilação de informações sobre ocupação, particularmente a dos pais, para fins de avaliação da mobilidade ocupacional intergeracional. O tema da mobilidade social tem sido pouco explorado na literatura nacional na área da saúde, merecendo maior atenção, já que se trata de um importante mecanismo de compensação e restrição das desigualdades sociais em saúde ${ }^{7}$. Além disso, a utilização de variáveis mais relacionadas ao poder de compra implica em grandes desafios acerca de sua padronização e possibilidade de comparação entre populações tão distintas.

A variável "número de bens" foi utilizada para gerar um indicador, o "índice de bens”, que considera que quanto mais rara for a presença de um bem na amostra, maior o seu "valor" 4 . Esta decisão parece interessante se considerarmos que o construto subjacente a ser apreendido é algo que seria uniformemente desejável 
por todos da população. Por outro lado, não considera as diferenças inerentes, e determinadas socialmente, acerca do que promove prestígio nos diferentes segmentos da população.

Uma das variáveis mais interessantes avaliadas na PMS foi a auto-avaliação da saúde ${ }^{3,4}$. A opção da PMS foi utilizar uma forma de questionamento absoluta ("Em geral, como o(a) senhor(a) avalia sua saúde atualmente?"), com respostas variando de muito ruim a muito boa em uma escala de 1 a $5^{8}$. Apesar desta forma de abordagem ser, ao que parece, a mais freqüentemente encontrada na literatura ${ }^{9}$, também tem sido comum o uso da pergunta comparativa (p.ex., "De um modo geral, em comparação com as pessoas da sua idade, como você considera o seu próprio estado de saúde?"). Há muito debate sobre qual das duas formas seria a mais adequada, alguns autores indicando que a pergunta comparativa teria a vantagem de "ajustar" pelo efeito idade e doenças a ela associadas ${ }^{9}$ e apresentar maior poder de predição de mortalidade ${ }^{10}$, pelo menos entre idosos do sexo masculino ${ }^{11}$. Por outro lado, alguns autores argumentam que, exatamente por ser "ajustada por idade”, a pergunta comparativa não é capaz de mensurar condições de saúde da mesma forma para diferentes grupos etários, característica essa que favoreceria o uso da pergunta não-comparativa em inquéritos em população com ampla variação etária e estudos longitudinais ${ }^{12,13}$. Entretanto, ao contrário do que se apregoava ${ }^{9}$, há indicações de que as medidas de auto-avaliação da saúde não são insensíveis à maneira como as perguntas são formuladas e parecem apreender diferentes dimensões da saúde percebida ${ }^{10,11,13,14}$. Neste sentido, o uso das duas versões, absoluta e comparativa, simultaneamente, tem sido sugerido como forma de auxiliar na elucidação dos diferentes construtos mapeados por cada uma delas, assim como para favorecer a comparabilidade entre resultados de diferentes estudos ${ }^{14}$.

Um outro ponto metodológico-operacional que merece destaque foi a utiliza- ção de um desenho amostral em três estágios, em que o processo de seleção dos domicílios (segundo estágio) foi realizado usando uma estratégia de amostragem inversa $^{15}$. Neste método, ao invés de se definir o número de domicílios a serem visitados para se conseguir as entrevistas, define-se o número de entrevistas realizadas considerando para fins de expansão da amostra o número de domicílios de fato visitados $^{15}$. Trata-se de uma estratégia mais eficiente para lidar com a não-resposta, sem haver necessidade de serem feitas correções no tamanho amostral ou para nãoresposta por ocasião do processo de expansão da amostra. Apesar deste inequívoco benefício em termos de eficiência estatística, vale salientar que ainda precisamos de ferramentas potentes para evitar os potenciais vieses ocasionados pela não-resposta. Em particular na PMS, mas um problema comumente observado em estudos de base populacional no Brasil, a recusa foi bem mais freqüente entre os de maior renda $(13,1 \%)$ quando comparada com o quinto de renda mais baixo $(3,0 \%)^{15}$. São várias as motivações que levam as pessoas a rejeitar participar em estudos, restando ao investigador buscar interpretações que sustentem (ou não) a possibilidade de que estes dados faltantes estejam sendo gerados de forma aleatória (pelo menos condicionalmente às variáveis observadas). Mesmo assim, tratam-se apenas de conjecturas, mas que poderiam ser mais convincentes se nossos estudos incorporassem mais freqüentemente elementos de análise de sensibilidade ${ }^{16}$.

Resultados interessantes acerca das desigualdades em saúde foram revelados pela PMS; dentre tantos, três me chamaram particular atenção. Primeiro, a identificação de que a saúde bucal, particularmente a perda de todos os dentes, pode ser um importante marcador da desigualdade. A PMS estimou que $14 \%$ dos brasileiros perderam todos os seus dentes naturais. O gradiente social para este indicador foi pronunciado, particularmente entre as mulheres com mais de 50 anos, em que $56 \%$ daquelas em pior condi- 
ção socioeconômica perderam todos os seus dentes contra $19 \%$ daquelas em melhor situação ${ }^{17}$. Neste sentido, pode vir a ser útil a incorporação de questões desse tipo, não só em estudos sobre o tema específico da saúde bucal, mas em investigações direcionadas para revelar e avaliar intervenções voltadas para a redução das desigualdades sociais em saúde. Segundo, a identificação de que a discriminação (por sexo, idade, pobreza, classe social, raça ou tipo de doença), pode ser um importante marcador da satisfação dos usuários com o atendimento de saúde ${ }^{18}$. Este achado tem implicações potenciais para as estratégias de organização do sistema de saúde no Brasil. Terceiro, o desvendamento de marcantes diferenças dos gastos entre os mais ricos e mais pobres. É mais uma importante faceta da desigualdade social o fato de que os mais pobres gastam $58 \%$ de sua renda com alimentação, e apenas 13\% e 5\% com saúde e educação, respectivamente. Entre os ricos, os gastos são de $25 \%$ com ali- mentação, $21 \%$ com saúde e $13 \%$ com educação. Ainda impressionante é o fato de que os medicamentos consomem $60 \%$ dos gastos em saúde dos mais pobres, percentual que cai para $21 \%$ entre os mais ricos. Os mais ricos, por outro lado, utilizam $40 \%$ dos seus gastos em saúde com planos de saúde, contra $4,3 \%$ dos mais pobres ${ }^{17}$.

A riqueza da PMS é tão grande que seria possível ainda aprofundar os debates sobre diversos outros aspectos metodológicooperacionais e sobre seus resultados. Claro que este não é objetivo deste texto. Aqui se pretendeu realizar um exercício sobre as implicações que determinadas decisões ou opções, muitas vezes estabelecidas sem que tenhamos a possibilidade de intervir, podem ter nos resultados de um estudo desta magnitude e importância. Não foi fácil exercitar o papel de crítico; trata-se de pesquisa exemplar que será por longo tempo um modelo inspirador para a epidemiologia nacional.

\section{Referências}

1. Szwarcwald CL, Bastos FI, Esteves MA. State of animus among Brazilians: influence of socioeconomic context? Cad Saude Publica 2005; 21(Suppl.1): 33-42.

2. Damacena GN, Vasconcellos MT, Szwarcwald CL. Perception of health state and the use of vignettes to calibrate for socioeconomic status: results of the World Health Survey in Brazil, 2003. Cad Saude Publica 2005; 21(Suppl.1): 65-77.

3. Theme-Filha MM, Szwarcwald CL, Souza-Junior PR. Socio-demographic characteristics, treatment coverage, and self-rated health of individuals who reported six chronic diseases in Brazil, 2003. Cad Saude Publica 2005; 21(Suppl.1): 43-53.

4. Szwarcwald CL, Souza-Junior PR, Esteves MA, Damacena GN, Viacava F. Socio-demographic determinants of selfrated health in Brazil. Cad Saude Publica 2005; 21 (Suppl.1): 54-64.

5. Romero DE, Leite IC, Szwarcwald CL. Healthy life expectancy in Brazil: applying the Sullivan method. Cad Saude Publica 2005; 21(Suppl.1): 7-18.

6. Wadsworth M. Early life. In: Marmot M \& Wilkinson RG (eds). Social Determinants of Health. Oxford: Oxford University Press; 1999. p. 44-63.
7. Bartley M, Plewis I. Does health-selective mobility account for socioeconomic differences in health? Evidence from England and Wales, 1971 to 1991. J Health Soc Behav 1997; 38(4): 376-86.

8. Szwarcwald CL, Leal MC, Gouveia GC, Souza WV. Desigualdades socioeconômicas em saúde no Brasil: resultados da Pesquisa Mundial de Saúde, 2003. Rev Bras Saude Mater Infant 2005, 5 (Suppl.1): S11-S22.

9. Idler EL, Benyamini Y. Self-rated health and mortality: a review of twenty-seven community studies. J Health Soc Behav 1997; 38(1): 21-37.

10. Heidrich J, Liese AD, Lowel H, Keil U. Self-rated health and its relation to all-cause and cardiovascular mortality in southern Germany. Results from the MONICA Augsburg cohort study 1984-1995. Ann Epidemiol 2002; 12(5): 338-45.

11. Manderbacka K, Kareholt I, Martikainen P, Lundberg O. The effect of point of reference on the association between self-rated health and mortality. Soc Sci Med 2003; 56(7): 1447-52.

12. Vuorisalmi M, Lintonen T, Jylha M. Global self-rated health data from a longitudinal study predicted mortality better than comparative self-rated health in old age. $J$ Clin Epidemiol 2005; 58(7): 680-7. 
13. Eriksson I, Undén AL, Elofsson S. Self-rated health Comparisons between three different measures. Results from a population study. Int J Epidemiol 2001; 30(2): 32633.

14. Baron-Epel O, Kaplan G. General subjective health status or age-related subjective health status: does it make a difference? Soc Sci Med 2001; 53(10): 1373-81

15. Vasconcellos MT, Silva PL, Szwarcwald CL. Sampling design for the World Health Survey in Brazil. Cad Saude Publica 2005; 21(Suppl.1): 89-99.
16. Greenland S. Basic methods for sensitivity analysis of biases. Int J Epidemiol 1996; 25(6): 1107-16

17. Szwarcwald CL, Viacava F, Vasconcellos MTL, Leal MC, Azevedo LO, Queiroz RSB, et al. Pesquisa Mundial de Saúde 2003: O Brasil em Números. RADIS/FIOCRUZ 2004; 23: 14-33.

18. Gouveia GC, Souza WV, Luna CF, Souza-Júnior PR, Szwarcwald CL. Health care users' satisfaction in Brazil, 2003. Cad Saude Publica 2005; 21(Suppl.1): 109-18. 